\title{
Railscan: A Tool for the Detection and Quantification of Rail Corrugation
}

\author{
Rui Gomes ${ }^{1}$, Arnaldo Batista ${ }^{1}$, Manuel Ortigueira ${ }^{1}$, \\ Raul Rato ${ }^{1}$, and Marco Baldeiras ${ }^{2}$ \\ ${ }^{1}$ Department of Electrical Engineering, Universidade Nova de Lisboa, Portugal \\ agb@fct.unl.pt \\ ${ }^{2}$ Refer, Rede Ferroviária Nacional, EP \\ mlbaldeiras@refer.pt
}

\begin{abstract}
Rail corrugation is a phenomenon that leads to a waving in the rails with wavelengths typically between $3 \mathrm{~cm}$ and $100 \mathrm{~cm}$ and amplitude levels of several microns. The genesis of this waving is complex. Rail corrugation is a recognized problem that leads to excess vibration on the rails and vehicles to a point of reducing their life span and compromising safety. In urban areas excess vibration noise is also a problem. A software tool was developed to analyze accelerometer signals acquired in the boggies of rail vehicles in order to quantify the rail corrugation according to their frequency and amplitude. A wavelet packet methodology was used in this work and compared with the One Third Octave Filter (OTOF) power representations, which is currently used in the industry. It is shown that the former produces better results.
\end{abstract}

Keywords: Rail Corrugation, Wavelets, Time-Frequency.

\section{Introduction}

Rail corrugation is a problem extensively felt by railway companies. This phenomenon is due to the railway traffic conditions that produce corrugation wavelengths in the railhead between $3 \mathrm{~cm}$ and $100 \mathrm{~cm}$ [1]. These rail irregularities are a matter of concern due to excess vibration loads in the vehicles and noise. Vibration may compromise safety and reduce the life span of the equipment, therefore requiring some type of rail maintenance. Early detection of the phenomenon to improve rail security and for economical reasons is a desired goal.

Corrugation measurement may be done using the direct and the indirect approach [2], [3]. In this work an indirect measurement approach in which the corrugation levels are obtained through the signals from axle-box accelerometers [3].

In this paper is presented a software tool, RailScan that integrates under the same roof the necessary signal processing steps and procedures for the rail corrugation detection and quantification using the EN ISO 3095 parameters, exploring the wavelet's superior ability for non-stationary signal analysis.

The RailScan user interface is designed to allow for the system parameters to be modified and adjusted for research purposes. The results are presented in a series of interactive results representations. The results numerical values may be exported for 
later comparative analysis. The software has been developed in Matlab [4]. RailScan corrugation signal analysis includes: 1 . Time-scale representation with user selected wavelets; 2. Base-line removal; 3. Mouse driven feature selection in the Time-Scale plan; 4. Wavelet-Packet implementation that results in power spectrum in the corrugation wavelengths, rail corrugation localization in the rail and its signal recovering in selected wavelet nodes; 5 .One Third Octave Filter (OTOF) power representations.

\section{Contribution to Technological Innovation}

Wavelet analysis is herein used as a tool for the analysis on the vibration signals due to the rail corrugation, following a new trend [1] in this research area. Wavelet analysis has been proved to be more adequate for the processing of non-stationary signals, such as these, for which the classical Fourier analysis presents limited results. However, the application of this tool lacks standardization procedures and overall validation. This work is a contribution for that goal, also being a preparation for the implementation of a version of the EN ISO 3095 with wavelets.

\section{Methods}

RailScan uses the Continuous Wavelet Transform (CWT) and the Wavelet Packet Transform, to analyze the axle-box accelerometer data. These methods will be described in this section. It should be emphasized that the CWT is used in the RailScan interface with a mouse driven selection tool to analyze user elected signal details, which is not represented were for lack of space.

\section{A. Continuous Wavelet Transform}

The Continuous Wavelet Transform is used in this work to perform time-scale analysis of corrugation signals. This is done multiplying a signal $x(t)$ by scaled and translated versions of $\psi(\tau)$ the mother wavelet:

$$
C W T_{x}^{\psi}(\tau, s)=\frac{1}{\sqrt{|s|}} \int x(t) \psi^{*}\left(\frac{t-\tau}{s}\right) d t .
$$

CWT is a function of two variables, the scale $s$ and position $\tau$ which is related with the localization of the Wavelet, as the wavelet is shifted through the signal. The scale parameter can be seen as a scale in a map, larger scale show global views and smaller scales show detailed information of a hidden pattern in a signal [5]. A time-scale signal representation is obtained where features are exposed and localized both in time and in the frequency, since scale can be converted to frequency. RailScan interface allows for the user to mouse select features in the time-scale plan and then automatically to synthesize the underling signal using Wavelet Packets, within the selected frequency boundaries. The synthesized signal may be the corrugation component which has been recovered from the whole signal, for analysis purposes. In this work it was found that the application of the complex Morlet wavelet gives better results for the real corrugation tested signals. 


\section{B. Wavelet Packets}

The CWT is generally a representation with a high degree of redundancy that doesn't allow for signal reconstruction, but permits time-scale signal representations with a user defined frequency resolution. However, the Discrete Wavelet Transform (DWT) allows for multiresolution signal reconstruction, although its time-scale representation being defined for consecutive frequencies that are a power of two related, in consecutive levels. This constraint may be avoided if the Wavelet Packet Transform (WPT) is used. The general representation of the wavelet tree of a second order, three levels Wavelet Packet tree is shown in Figure 1. The filters sequence has been altered in order to give the last tree nodes (the tree leaves) a frequencial order. The last line numbers sequence represents the nodes natural order. $\mathrm{A}_{1}$ stands for signal approximation in level 1 due to the Low Pass (LP) filtering, and $\mathrm{D}_{1}$ stands for signal detail in level 1 due to the High Pass (HP) filtering level 1. Likewise $\mathrm{DA}_{2}$, for instance stands for the detail of approximation signal $\mathrm{A}_{1}$. For the other nodes the same rule is applied.

The leaves frequency nodes progress linearly between zero and the Nyquist frequency, a feature that makes the Wavelet Packet transform a desirable tool in this application. The frequency resolution may be increased, and is limited by the signal length. Of course time resolution decreases in the leave's nodes, as frequency resolution increases.

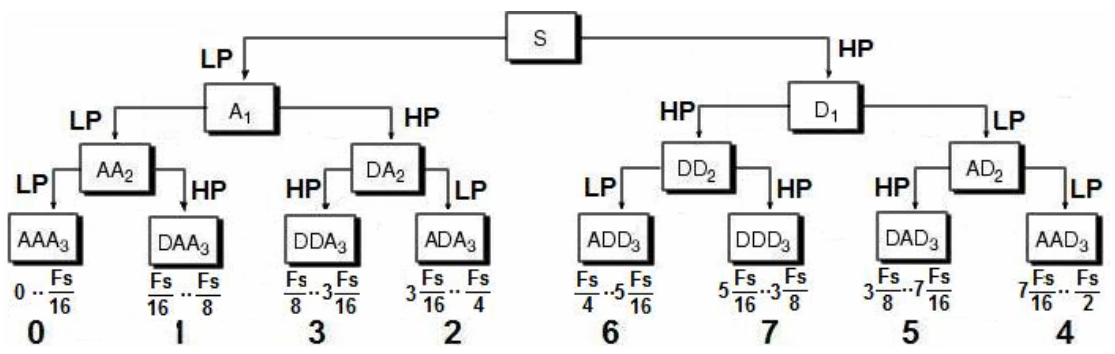

Fig. 1. Wavelet tree with level 3 decomposition (in the frequencial order)

A scheme was used with 64 leaves which cover the corrugation wavelength band with enough resolution. For this a six level WPT is implemented with a precision of $3.9 \mathrm{~Hz}$. Applying expression (7) with $v=1 \mathrm{~m} / \mathrm{s}$ the wavelength scale is obtained as shown in Fig. 3 (horizontal axis). The Daubechies D10 wavelet was used [5].

\section{One Third Octave Filter (OTOF)}

The European Standard EN ISO 3095 [2] was followed in this work, regarding the indirect measurement of the corrugation level. This chapter includes the case of the data being acquired with an axle-box accelerometer. Accordingly, the OTOF is used to identify the frequencies contents of the vibration due to corrugation, in a predefined scale [2] shown in the first column of Table 1. This procedure will allow for result comparison between the classical OTOF spectra and the one derived from Wavelet Packets application. 
A series of band-pass filters for each central frequency $\left(f_{c}\right)$ are defined, for each the lower cutoff frequency $\left(f_{\text {lcut }}\right)$ and higher cutoff frequency $\left(f_{\text {hcut }}\right)$ is give by the expressions:

$$
\begin{gathered}
f_{\text {lcut }}=\frac{1}{10^{\frac{1}{20}} \cdot f_{c}} . \\
f_{\text {hcut }} t=10^{\frac{1}{20}} \cdot \mathrm{f}_{\mathrm{c}} . \\
f=\frac{v}{\lambda} .
\end{gathered}
$$

Using expression (7) the pre-defined ISO wavelengths (for $v=1 \mathrm{~m} / \mathrm{s}$ ) were converted to frequencies. Third order Butterworth filters are defined as in [6]. Table 1 contains

\begin{tabular}{|c|c|c|}
\hline $\begin{array}{c}\text { Wavelength }(\mathrm{m}) \\
\lambda\end{array}$ & $\begin{array}{c}\text { Central frequency }(\mathrm{Hz}) \\
\boldsymbol{f}_{\boldsymbol{c}}\end{array}$ & $\begin{array}{l}\text { One third octave band (Hz) } \\
\boldsymbol{f}_{\boldsymbol{l} \boldsymbol{c u t}}-\boldsymbol{f}_{\boldsymbol{h} \boldsymbol{c u t}}\end{array}$ \\
\hline 0.63 & 1.5873 & $1.4147-1.7810$ \\
\hline 0.5 & 2.0 & $1.7825-2.2440$ \\
\hline 0.4 & 2.5 & $2.2281-2.8050$ \\
\hline 0.315 & 3.1746 & $2.8294-3.5620$ \\
\hline 0.25 & 4.0 & $3.5650-4.4881$ \\
\hline 0.2 & 5.0 & $4.4563-5.6101$ \\
\hline 0.16 & 6.25 & $5.5703-7.0126$ \\
\hline 0.125 & 8.0 & $7.1300-8.9761$ \\
\hline 0.1 & 10.0 & $8.9125-11.2202$ \\
\hline 0.08 & 12.5 & $11.1406-14.0252$ \\
\hline 0.063 & 15.8730 & $14.1468-17.8098$ \\
\hline 0.05 & 20.0 & $17.8250-22.4404$ \\
\hline 0.04 & 25.0 & $22.2813-28.0505$ \\
\hline 0.0315 & 31.7460 & $28.2937-35.6196$ \\
\hline 0.025 & 40.0 & $35.6500-44.8807$ \\
\hline 0.02 & 50.0 & $44.5625-56.1009$ \\
\hline 0.016 & 62.5 & $55.7032-70.1262$ \\
\hline 0.00125 & 80.0 & $71.3001-89.7615$ \\
\hline 0.001 & 100.0 & $89.1251-112.2018$ \\
\hline 0.008 & 125.0 & $111.4064-140.2523$ \\
\hline 0.0063 & 158.7302 & $141.4684-178.0982$ \\
\hline 0.005 & 200.0 & $178.2502-224.4037$ \\
\hline 0.004 & 250.0 & $222.8127-280.5046$ \\
\hline 0.00315 & 317.4603 & $282.9368-356.1963$ \\
\hline
\end{tabular}
the OTOF bands for the 23 steps according to [6].

Table 1. One third octave band structure

For frequencies close to zero or the Nyquist value an interpolation factor is applied to the data to improve filter stability. For each band the resulting filtered signal power is obtained to be compared with the pre-defined ISO power values [2]. Following the 


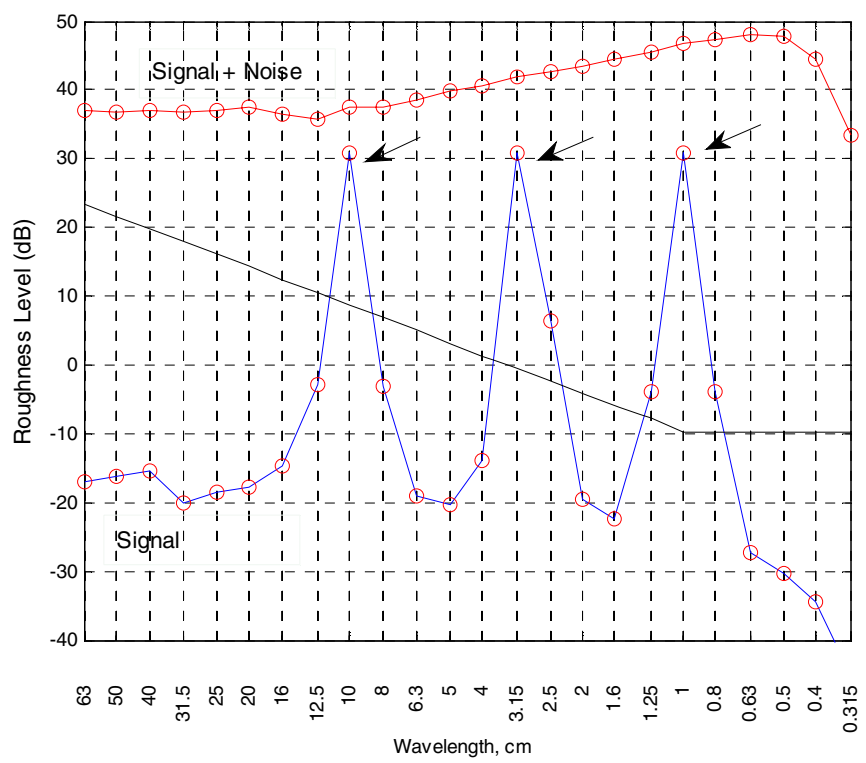

Fig. 2. Power Spectrum of the Roughness Level, 1/3 Octave Power Spectrum for simulated signal and simulated sinal added to noise

mentioned ISO rule the rail roughness limit spectrum is represented in Fig. 2 by the solid line.

\section{Pre Processing}

For the wavelet processing, the higher wavelength band extends to infinity. Since the corrugation signal may contain higher energetic long wavelengths components that would result in power peaks, which would hide the power in the interest bands, a base line removal step is required. Having in account that the higher ISO wavelength value is $0.63 \mathrm{~m}$ and the long wavelength corrugation extends to $1.00 \mathrm{~m}$ [1], the data is filtered with a wavelet packet filter to remove signal components with wavelengths higher than $1.00 \mathrm{~m}$.

The wavelet packet filter was implemented using the reconstruction of the signal associated to the few base-line nodes and then subtracting this residue from the original corrugation signal. Since only a few nodes are involved, the resulting algorithm is rather fast. Moreover, the wavelet packet frequency structure allows for a flexible selection of the cutoff frequency value.

\section{Application to a Simulated Signal}

A simulated corrugation signal with three sinusoids corresponding to a $1000 \mathrm{~m}$ rail section, with sampling frequency of $500 \mathrm{~Hz}$ at an average speed of $1 \mathrm{~m} / \mathrm{s}$ is obtained:

$$
y(n)=A\left[\operatorname{sen}\left(2 \pi f_{1} n_{1}\right)+\operatorname{sen}\left(2 \pi f_{2} n_{2}\right)+\operatorname{sen}\left(2 \pi f_{3} n_{3}\right)\right] \times \mathrm{N}
$$




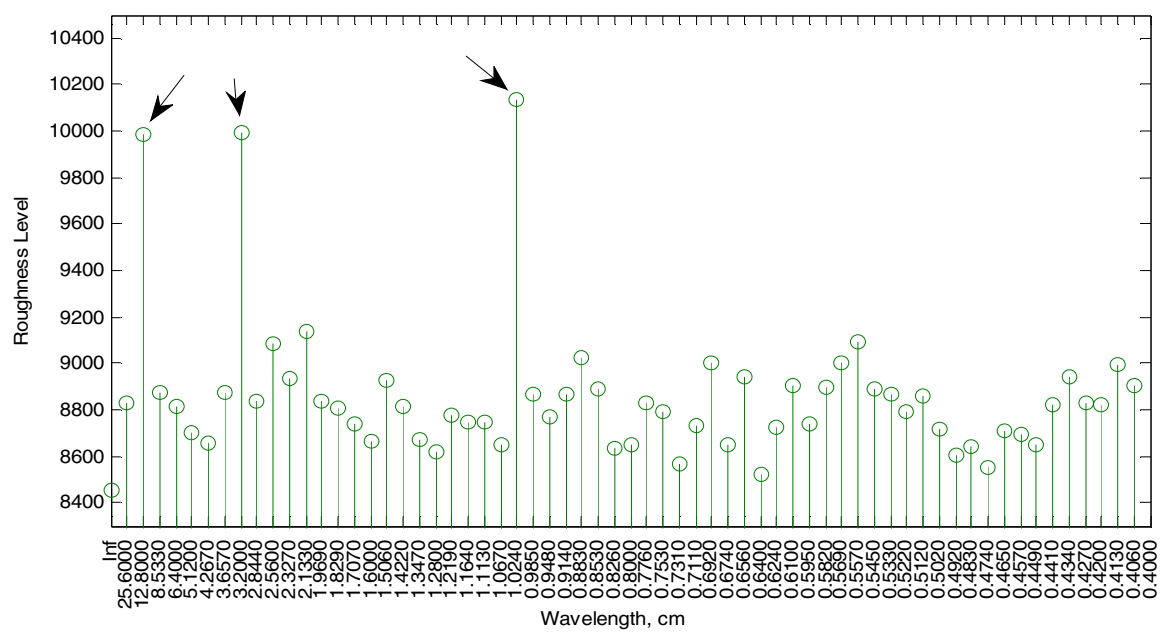

Fig. 3. Power Spectrum of the Roughness Level, Wavelet Packet Spectrum for signal + noise

With $f_{1}$ corresponding to $10 \mathrm{~Hz}(\lambda=10 \mathrm{~cm}), f_{2}$ corresponding to $33.33 \mathrm{~Hz}(\lambda=3 \mathrm{~cm})$ and $f_{3}$ corresponding to $100 \mathrm{~Hz}(\lambda=1 \mathrm{~cm}), n_{1} \in[1,125000], n_{2} \in[187500,312500]$, $n_{3} \in[375000,500001]$ and $A=100 \mu \mathrm{m}$ being the corrugation amplitude value. A random noise $N$ with zero mean and standard deviation of $150 \mu \mathrm{m}$ is added. Fig. 2 shows that the noise added signal in the $O T O F$ power spectrum no longer shows the signal components (arrow marked) whereas in fig. 3 plot (WPT) the peaks are clearly detected (arrow marked).

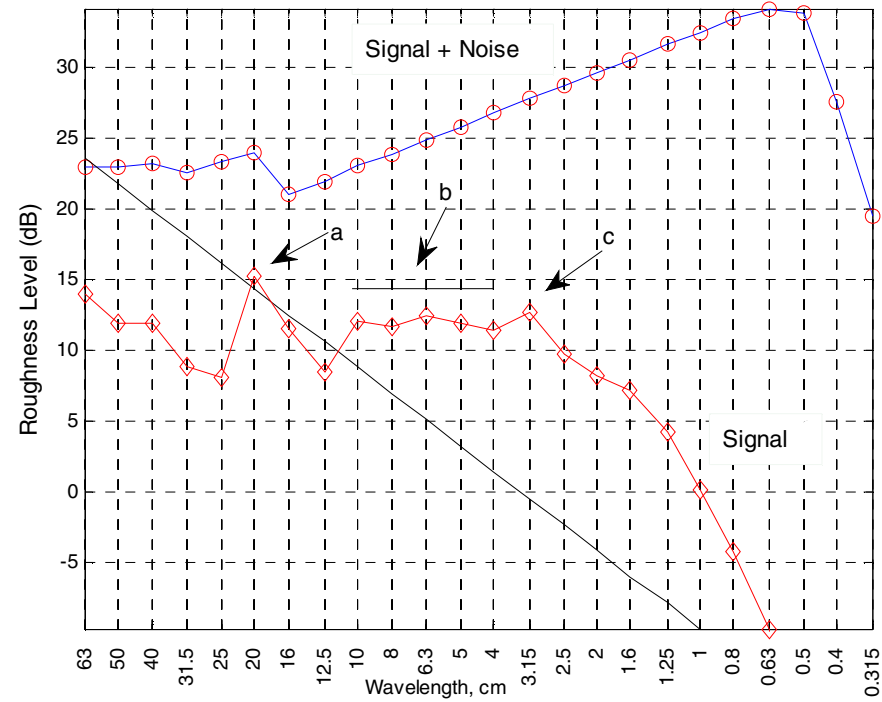

Fig. 4. Power Spectrum of the Roughness Level, 1/3 Octave Power Spectrum 


\section{Application to a Corrugation Signal}

Fig. 4 shows the OTOF power spectrum of a real corrugation signal and the spectrum of the same signal added to a simulated noise of standard deviation of $135 \mu \mathrm{m}$. The noisy signal spectrum no longer detects the relevant components $b$ and $c$. However the WPT plot, in Fig. 5, detects components $b$ and $c$, with component $a$ clearer defined. This shows the superior detection ability of the WPT algorithm.

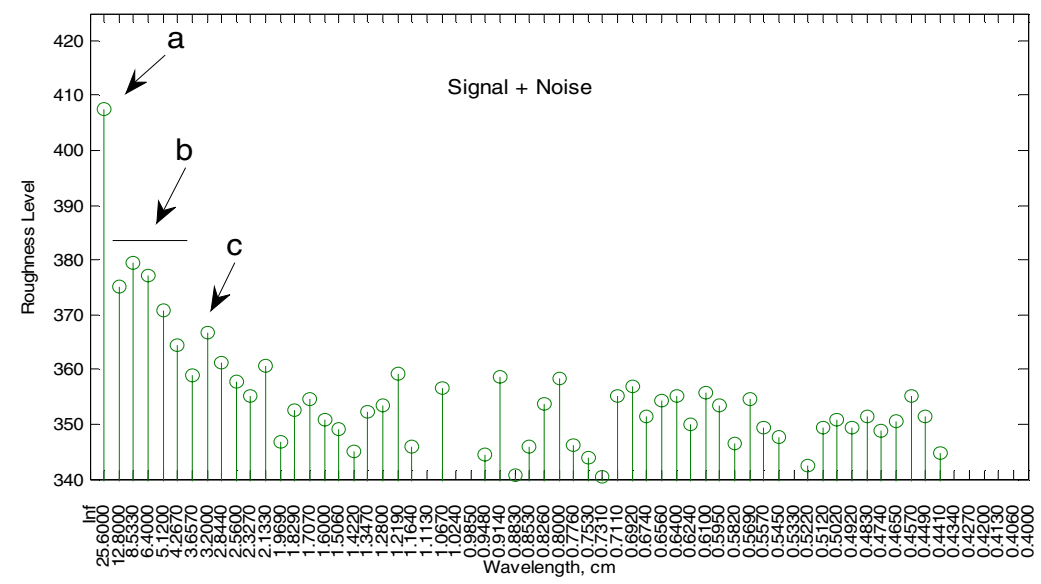

Fig. 5. Power Spectrum of the Roughness Level, Wavelet Packet Spectrum for signal + noise

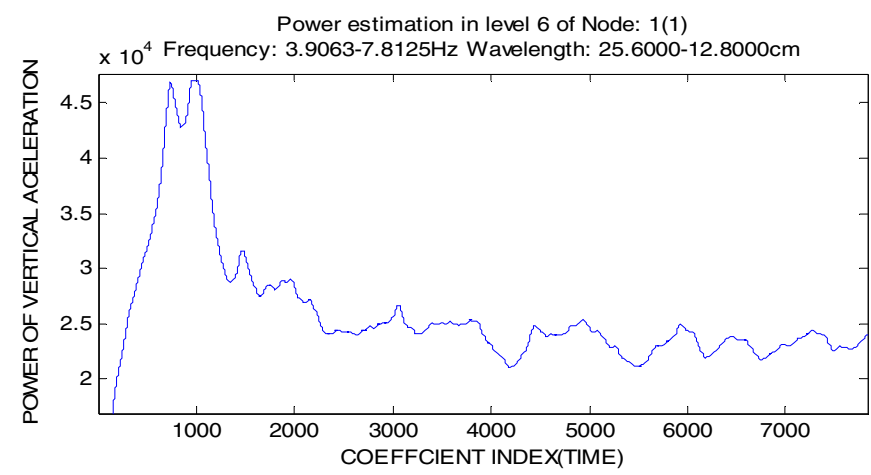

Fig. 6. Wavelet Coefficient Power estimation showing that excess corrugation in the wavelength $25.6-12.8 \mathrm{~cm}$ band exists, in the rail track between 61 and $157 \mathrm{~m}$, see text

\section{Corrugation Spatial Localization}

One of the advantages of the Wavelet Transform is the ability of the time localization along with the frequency (scale) description. This feature is used in the WPT to locate in the rail, the places where corrugation in a particular wavelength occurs [7], an information important for railway companies that will then perform some type of remediation procedures [8]. Fig. 6 shows that corrugation power in the wave-length 
band $25.6-12.8 \mathrm{~cm}$ is higher between wavelet coefficients 481 and 1226 which corresponds to the rail track between 61 and $157 \mathrm{~m}$.

\section{Conclusions and Further Developments}

Wavelet analysis has produced promising results both for simulated data and corrugation detection for accelerometer signals acquired in the axle-box of a railway vehicle. Wavelets outperform the classical OTOF method for signals contaminated with high levels of noise. This feature seems important in the development of portable invehicle corrugation detectors as well as improved accelerometer data analysis. For future work it is planned to represent wavelet analysis results in the EN ISO 3095 representation, as well as address issues of standardization and referencing.

Acknowledgements. The collaboration of Dr. Stuart Grassie of Railmeasurement in this work is acknowledge.

\section{References}

1. Grassie, S.L.: Measurement of railhead longitudinal profiles: a comparison between different techniques. Wear 191, 245-251 (1996)

2. prEN ISO 3095: Railway applications - Acoustics - Measurements of noise emitted by railbound vehicles

3. Verheijen, E.: E, A survey on roughness measurements. Journal of Sound and Vibration 293, 784-794 (2006)

4. Matworks Inc., Natick, MA 01760-2098

5. Daubechies, I.: Ten Lectures on Wavelets, Society for Industrial and Applied Mathematics

6. ANSI/ASA S1.11-1986(R1998) American National Standard Specifications for OctaveBand and Fractional-Octave-Band Analog and Digital Filters

7. Caprioli, A., Cigada, A., Raveglia, D.: Rail inspection in track maintenance: a benchmark between the wavelet approach and the more conventional Fourier analysis. Mechanical System and Signal Processing 21, 631-652 (2007)

8. Grassie, S.L.: Rail Corrugation: advances in measurement, understanding and treatment. Wear 258, 1224-1234 (2005) 